\title{
Erratum: Searching for flavored gauge bosons
}

\author{
Eung Jin Chun, ${ }^{a}$ Arindam Das, ${ }^{a}$ Jinsu $\mathrm{Kim}^{b, 1}$ and Jongkuk Kim ${ }^{c}$ \\ ${ }^{a}$ Korea Institute for Advanced Study, Seoul 02455, Korea \\ ${ }^{b}$ Institute for Theoretical Physics, Georg-August University Göttingen, \\ Friedrich-Hund-Platz 1, Göttingen D-37077, Germany \\ ${ }^{c}$ Department of Physics, BK21 Physics Research Division, Institute of Basic Science, \\ Sungkyunkwan University, Suwon 440-746, Korea \\ E-mail: ejchun@kias.re.kr, arindam@kias.re.kr, \\ jinsu.kim@theorie.physik.uni-goettingen.de, jongkukkim@skku.edu
}

ERRATUM TO: JHEP02(2019)093

ARXIV EPRINT: 1811.04320

We provide a change to the LHC bounds on $B-3 L_{i}(i=1,2,3)$ scenario in ref. [1]. In figure 3 of the original article [1], the LHC bounds on $B-3 L_{i}$ are depicted as solid and dashed brown curves. They are modified as figure 1 in this erratum.

Accordingly the last paragraph of section 7 of the original article [1] is updated as follows. For all $B-3 L_{i}$ cases, the LHC bounds become the strongest bounds for large $M_{Z^{\prime}}$ region; $M_{Z^{\prime}} \gtrsim 150 \mathrm{GeV}$ for $i=1,2$ cases and $M_{Z^{\prime}} \gtrsim 500 \mathrm{GeV}$ for $i=3$ case. In the $B-3 L_{1}$ case, the LEP bounds on $e^{-} e^{+} \rightarrow \ell^{-} \ell^{+}$processes give the most stringent bounds on small $M_{Z^{\prime}}$ region, $10 \mathrm{GeV} \lesssim M_{Z^{\prime}} \lesssim 150 \mathrm{GeV}$. The $B-3 L_{2}$ case is particularly interesting. Due to the strong bounds from HFAG lepton universality test, only a small region $60 \mathrm{GeV} \lesssim M_{Z^{\prime}} \lesssim 150 \mathrm{GeV}$ is consistent with all the constraints at $2 \sigma$.

\section{Acknowledgments}

We thank Hongkai Liu for pointing out our mistakes.

\footnotetext{
${ }^{1}$ Corresponding author.
} 

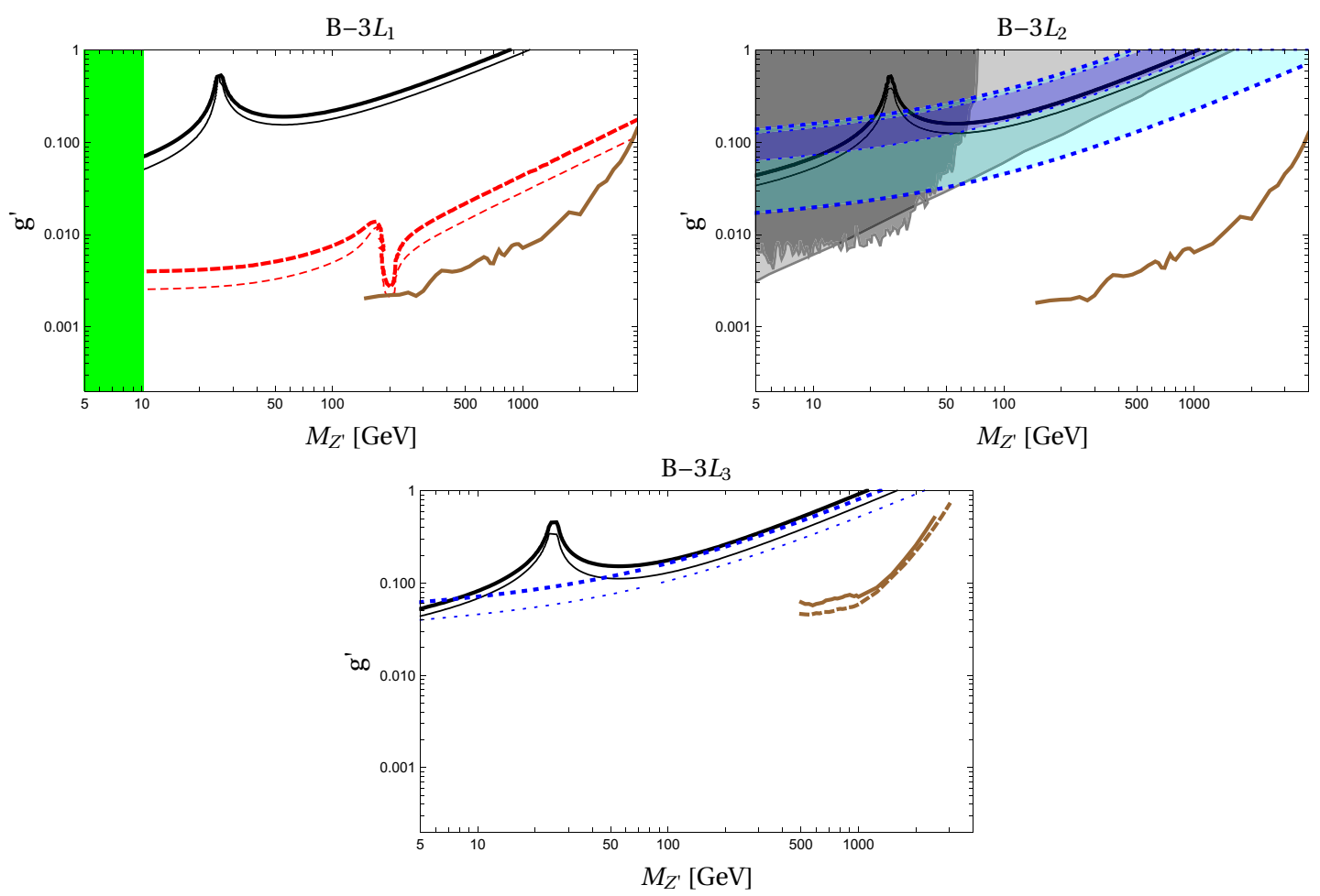

Figure 1. Upper left panel: bounds on $g^{\prime}$ and $M_{Z^{\prime}}$ for the $B-3 L_{1}$ model. The thin (thick) dashedred and solid-black curves correspond to $1 \sigma(2 \sigma)$ bounds from LEP search [2] and SLD/LEP Z-decay lepton universality test [3], respectively. The green region is excluded by BaBar bounds [4]. The brown curve is the LHC bound from ATLAS observed limits. Upper right panel: bounds on $g^{\prime}$ and $M_{Z^{\prime}}$ for the $B-3 L_{2}$ model. The thin (thick) solid-black curve corresponds to $1 \sigma(2 \sigma)$ bound from the SLD/LEP $Z$-decay lepton universality test [3]. The blue (cyan) shaded region between thin (thick) dotted-blue curves is the $1 \sigma(2 \sigma)$ allowed region by the HFAG lepton universality test [5]. The brown curve is the LHC bound from ATLAS observed limits. The lighter- and darker-grey regions are excluded by neutrino-trident bound [6,7] and the LHC bound for $Z \rightarrow 4 \mu$ [8]. Therefore, only a small region $60 \mathrm{GeV} \lesssim M_{Z^{\prime}} \lesssim 150 \mathrm{GeV}$ is consistent with all the constraints at $2 \sigma$. Lower panel: bounds on $g^{\prime}$ and $M_{Z^{\prime}}$ for the $B-3 L_{3}$ model. The thin (thick) dotted blue and solid black curves correspond to $1 \sigma(2 \sigma)$ bounds from the HFAG lepton universality test [5] and SLD/LEP $Z$-decay lepton universality test [3], respectively. The solid and dashed brown curves are the LHC bounds from ATLAS and CMS respectively.

Open Access. This article is distributed under the terms of the Creative Commons Attribution License (CC-BY 4.0), which permits any use, distribution and reproduction in any medium, provided the original author(s) and source are credited.

\section{References}

[1] E.J. Chun, A. Das, J. Kim and J. Kim, Searching for Flavored Gauge Bosons, JHEP 02 (2019) 093 [arXiv: 1811.04320] [inSPIRE].

[2] ALEPH, DELPHI, L3, OPAL collaborations and LEP Electroweak Working Group, Electroweak Measurements in Electron-Positron Collisions at W-Boson-Pair Energies at LEP, Phys. Rept. 532 (2013) 119 [arXiv:1302.3415] [INSPIRE]. 
[3] ALEPH, DELPHI, L3, OPAL, SLD collaborations, LEP Electroweak Working Group, SLD Electroweak Group and SLD Heavy Flavour Group, Precision electroweak measurements on the $Z$ resonance, Phys. Rept. 427 (2006) 257 [hep-ex/0509008] [INSPIRE].

[4] BABAr collaboration, Search for a Dark Photon in $e^{+} e^{-}$Collisions at BaBar, Phys. Rev. Lett. 113 (2014) 201801 [arXiv:1406 . 2980] [INSPIRE].

[5] HFLAV collaboration, Averages of b-hadron, c-hadron and $\tau$-lepton properties as of summer 2016, Eur. Phys. J. C 77 (2017) 895 [arXiv: 1612.07233] [INSPIRE].

[6] CCFR collaboration, Neutrino tridents and W Z interference, Phys. Rev. Lett. 66 (1991) 3117 [INSPIRE].

[7] W. Altmannshofer, S. Gori, M. Pospelov and I. Yavin, Neutrino Trident Production: A Powerful Probe of New Physics with Neutrino Beams, Phys. Rev. Lett. 113 (2014) 091801 [arXiv: 1406.2332] [INSPIRE].

[8] CMS collaboration, Search for an $L_{\mu}-L_{\tau}$ gauge boson using $Z \rightarrow 4 \mu$ events in proton-proton collisions at $\sqrt{s}=13 \mathrm{TeV}$, Phys. Lett. B 792 (2019) 345 [arXiv:1808.03684] [INSPIRE]. 